\title{
Silencing of PMEPA1 accelerates the growth of prostate cancer cells through AR, NEDD4 and PTEN
}

\author{
Hua Li ${ }^{1}$, Ahmed A. Mohamed ${ }^{1}$, Shashwat Sharad ${ }^{1}$, Elizabeth Umeda ${ }^{1}$, Yingjie Song ${ }^{1}$, \\ Denise Young ${ }^{1}$, Gyorgy Petrovics ${ }^{1}$, David G. McLeod ${ }^{1,2}$, Isabell A. Sesterhenn ${ }^{3}$, \\ Taduru Sreenath ${ }^{1}$, Albert Dobi ${ }^{1}$, Shiv Srivastava ${ }^{1}$ \\ ${ }^{1}$ Center for Prostate Disease Research, Department of Surgery, Uniformed Services University of the Health Sciences, \\ Bethesda, MD 20814, USA \\ ${ }^{2}$ Urology Service, Walter Reed National Military Medical Center, Bethesda, MD 20814, USA \\ 3 Joint Pathology Center, Silver Spring, MD 20910, USA \\ Correspondence to: \\ Albert Dobi, e-mail: adobi@cpdr.org \\ Shiv Srivastava, e-mail: ssrivastava@cpdr.org \\ Keywords: prostate cancer, PMEPA1, androgen receptor, NEDD4, enzalutamide \\ Received: December 31, $2014 \quad$ Accepted: March 09, $2015 \quad$ Published: March 30, 2015
}

\section{ABSTRACT}

Androgen Receptor (AR) is the male hormone receptor and a nuclear transcription factor which plays a central role in the growth of normal and malignant prostate gland. Our earlier studies defined a mechanistic model for male hormone dependent regulation of AR protein levels in prostate cancer (CaP) cells through a negative feedback loop between AR and PMEPA1, an androgen induced NEDD4 E3 ubiquitin ligase binding protein. This report focuses on the impact of PMEPA1 silencing on CaP biology. PMEPA1 knockdown accelerated the growth of CaP tumor cells in athymic nude mice. In cell culture models knockdown of PMEPA1 resulted in resistance to AR inhibitors enzalutamide and bicalutamide. While, AR protein down regulation by NEDD4 was PMEPA1 dependent, we also noted a PMEPA1 independent downregulation of PTEN by NEDD4. In a subset of human CaP, decreased PMEPA1 mRNA expression significantly correlated with increased levels of AR transcription target PSA, as a surrogate for elevated AR. This study highlights that silencing of PMEPA1 accelerates the growth of CaP cells through AR, NEDD4 and PTEN. Thus, the therapeutic restoration of PMEPA1 represents a promising complementary strategy correcting for AR and PTEN defects in CaP. Statement of significance: Here we define that silencing of PMEPA1 facilitates the growth of CaP cells and modulates AR through NEDD4 and PTEN. The restoration of PMEPA1 represents a promising complementary therapeutic strategy correcting for AR and PTEN defects.

\section{INTRODUCTION}

AR is a nuclear transcription factor that executes key function in normal and malignant growth of the prostate gland [1-5]. The tight control of AR signaling is critical in maintaining the homeostasis of the prostate gland. Malignancy of prostate still remains the most common cancer detected in American men and second leading cause of cancer related deaths $[6,7]$. Cancerassociated dysfunctions of AR, such as aberrant activation due to mutations, amplification, splicing and cross talk with other pro-cancer signaling pathways contribute to prostate cancer $(\mathrm{CaP})$ development and progression $[1-5,8]$. In clinical practice, androgen ablation continues to be the corner stone for the treatment of advanced CaP [7, 9-11]. Unfortunately, androgen ablation treated CaPs eventually become treatment resistant, the stage of the disease known as castration resistant $\mathrm{CaP}$ (CRPC). Altered AR expression or function (gain or loss) resulting from genomic or non-genomic mechanisms associate with CRPC [1-5]. In contrast to other aspects of AR alterations, only a few studies have addressed how AR protein turnover is altered in $\mathrm{CaP}[12,13]$. A shorter half-life of AR was noted in 
the androgen responsive LNCaP cells as compared to its longer half life in the androgen refractory derivative C4-2B cells [14]. Early reports of the mechanisms of AR degradation showed that MDM2 E3 ubiquitin ligase targeted the AR through AKT mediated phosphorylation of MDM2 [15]. Our studies have implicated NEDD4 E3 ligase in AR degradation [16-19]. Studies have also described downregulation of AR by proteasome dependent degradation during mitosis suggesting AR as a licensing factor for DNA replication in CaP cells [20, 21]. Emerging data also suggests for the role of other E3 ubiquitin ligases (CHIP, SKP2, Siah2, RNF6) in the AR degradation or transcriptional activation [13]. A recent report showed that SPOP E3 ubiquitin ligase degrades AR protein and the recurrent genomic mutations of the SPOP noted in 5-10\% of CaPs impair the SPOP mediated AR degradation [22, 23].

PMEPA1 was originally identified by our laboratory as a prostate abundant, highly androgen induced gene that mapped to chromosome 20q13.31-q13.33 [16]. Human PMEPA1 protein exhibits $83 \%$ amino acid identity to the mouse Nedd4-bindng protein, N4wbp4 $[16,24]$. PMEPA1 is a direct transcriptional target of $\mathrm{AR}$ in CaP cells [18]. Further Investigations discovered a PMEPA1-AR negative feedback loop in the regulation of AR protein levels in CaP cells [19]. Members of the NEDD4 family of proteins are E3 ubiquitin ligases, which catalyze degradation of target proteins of physiologically important functions by the ubiquitinprosteasome pathway $[25,26]$. Initial evaluations of PMEPA1 mRNA expression in matched normal and prostate tumor specimens suggested decreased expression of PMEPA1 in two-third of CaP patients [17]. In contrast to CaPs, higher expression of PMEPA1 has been noted in multiple solid tumors $[27,28]$. Studies have also shown induction of PMEPA1 expression by transforming growth factor- $\beta$ (TGF- $\beta$ ) that was associated with colonocyte terminal differentiation [29]. Subsequent studies have defined that PMEPA1 inhibits TGF- $\beta$ receptor 1 meditated signaling through a negative feedback loop by sequestering R-Smads [30]. Increased $P M E P A 1$ expression in breast and lung cancer may lead to inhibition of TGF- $\beta$ signaling [31-33]. PMEPA1 has also been reported to promote the proliferation of AR negative CaP cells, PC3, through the Smad3-4/CMYC/p21Cip1 pathway [34, 35]. Taken together, both reduced and increased $P M E P A 1$ expression may promote tumorigenesis through distinct cell signaling pathways in a given cellular background.

In this report, we present new findings on the cancer biologic properties of decreased PMEPAI expression. Loss or decreased PMEPAI expression in $\mathrm{CaP}$ contributes to accelerated cell growth through increased AR and NEDD4, decreased PTEN levels and confers resistance to AR inhibitors used in androgen ablation therapy.

\section{RESULTS}

\section{Inhibition of PMEPA1 promotes the growth of prostate cancer cells}

We have examined the impact of PMEPAI depletion on tumor growth in vivo. We have characterized PMEPA1shRNA-LNCaP and PMEPA1shRNA-VCaP stable transfectants to assess the growth of prostate tumor cells in vitro. We observed enhanced cancer cell biologic features by monitoring cell growth, BrdU incorporation, cell plating efficiency, soft agar colony formation, growth response to androgen and cell cycle (Supplementary Figure 1).

We evaluated the tumor growth characteristics of PMEPA1shRNA harboring LNCaP cells in athymic nude mouse tumor xenograft model. The PMEPA1shRNALNCaP and control shRNA-LNCaP cells began to form subcutaneous tumors at 4 weeks post-injection. The overall growth of PMEPA1shRNA-LNCaP derived tumors was significantly faster than control derived tumors $(p<0.05)$ (Figure 1A). At 9 week post-injection among 20 mice in each group, 18 mice formed measurable subcutaneous tumors in the PMEPA1shRNA-LNCaP group. In contrast, only 12 mice formed tumors in the control group. The tumor formation rate was $90 \%$ in the PMEPA1shRNA-LNCaP group and only $60 \%$ in the control. The average tumor volume was $2246.04 \mathrm{~mm}^{3}$ for PMEPA1shRNA-LNCaP and $1211.64 \mathrm{~mm}^{3}$ for the control. PMEPA1shRNA-LNCaP tumors expressed higher levels of AR and PSA proteins compared to control cells as assessed by immunohistochemistry (IHC) (Figure 1B and Table 1). The tumor size in the control group decreased $21 \%$ at two weeks post-injection in response to castration. In contrast, PMEPA1shRNA-LNCaP xenografts were refractory to castration. By seven weeks post-castration, the overall tumor size was four times larger than the initial tumor size $(100 \%)$ in PMEPA1shRNA harboring xenografts (increased by $304 \%$ ), in contrast to the control group (increased by 69\%) $(p<0.05)$ (Figure 1C). Taken together, both in vitro and in vivo growth characteristics of PMEPA1shRNA harboring cells resulted in increased AR, gain of AR function and accelerated tumor growth under normal, as well as under castrate conditions.

\section{PMEPA1 depletion leads to resistance to AR inhibitors}

To further investigate the role of decreased PMEPA1 levels in enhancing tumor cell growth by gain of AR function, dose and time kinetic response to the AR inhibitors, enzalutamide and bicalutamide were assessed in cell growth assays. PMEPA1 depletion conferred resistance to AR inhibitors in both $\mathrm{LNCaP}$ and $\mathrm{VCaP}$ cells (Figure 2A and 2B, Supplementary Figure 2A and 2B). Additionally, enhanced resistance to AR inhibitors was confirmed by BrdU incorporation, soft agar colony formation and 


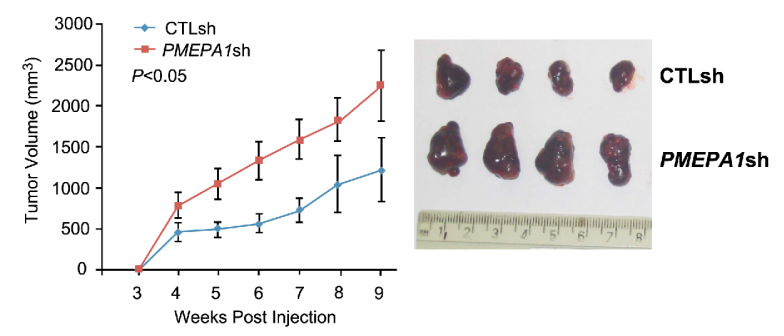

B
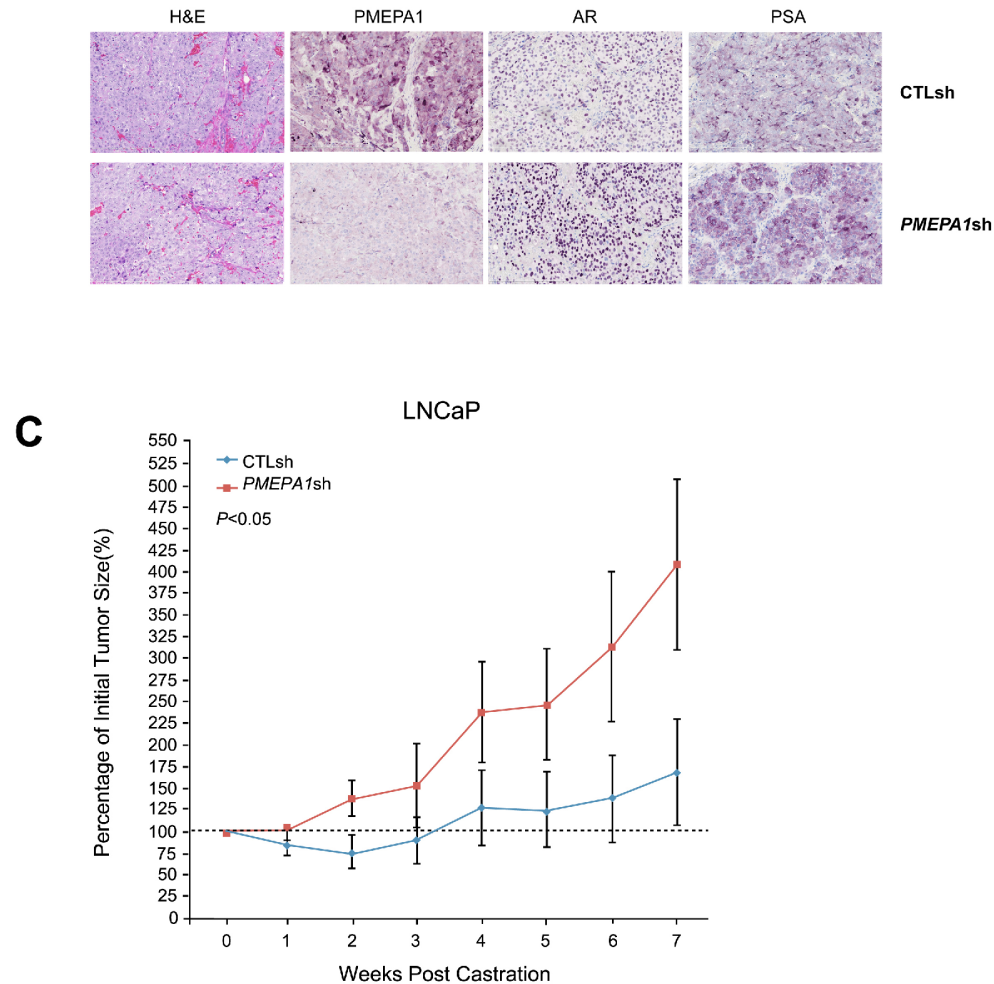

Figure 1: Inhibition of PMEPA1 promotes the growth of prostate cancer cells in vivo. (A) LNCaP cells harboring PMEPA1shRNA form larger volume of xenografts in athymic nude mice compared to control shRNA (CTLsh) LNCaP cells (graph and photograph of representative tumors; $p<0.05$ ). (B) Elevated levels of AR and PSA are detected in tumors of PMEPA1shRNA expressing LNCaP cells by immunohistochemistry (representative IHC images at 20X magnification). (C) Castration attenuates the growth of LNCaP tumors in vivo, in contrast, PMEPA1shRNA harboring tumors continue to grow at accelerated rate $(p<0.05)$.

Table 1: Higher expression level of AR and PSA were detected in PMEPA1shRNA-LNCaP xenografts with IHC staining

\begin{tabular}{l|c|c|c|}
\hline \multicolumn{1}{l}{ LNCaP xenografts } & \multicolumn{3}{c}{ Area (\%) of positive cells } \\
\cline { 2 - 4 } \multicolumn{1}{c|}{} & PMEPA1 & AR & PSA \\
\hline CTLsh & $37.13 \pm 1.76$ & $12.57 \pm 3.32$ & $7.02 \pm 1.20$ \\
\hline PMEPA1sh & $2.86 \pm 0.70$ & $32.58 \pm 1.46$ & $17.65 \pm 2.49$ \\
\hline$P$ value & $<0.01$ & $<0.01$ & $<0.01$ \\
\hline
\end{tabular}


A

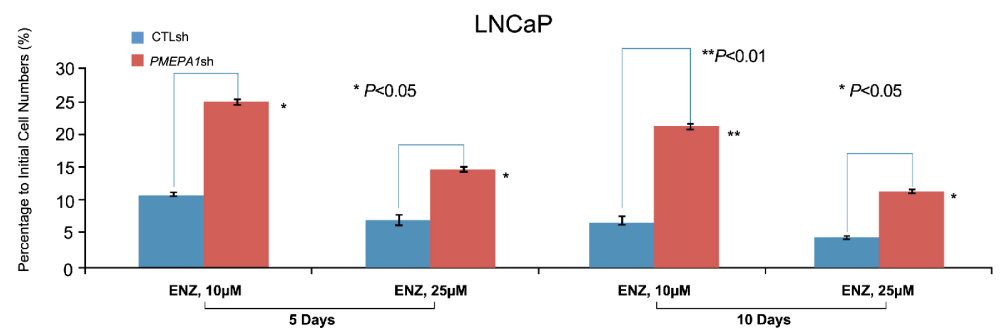

B

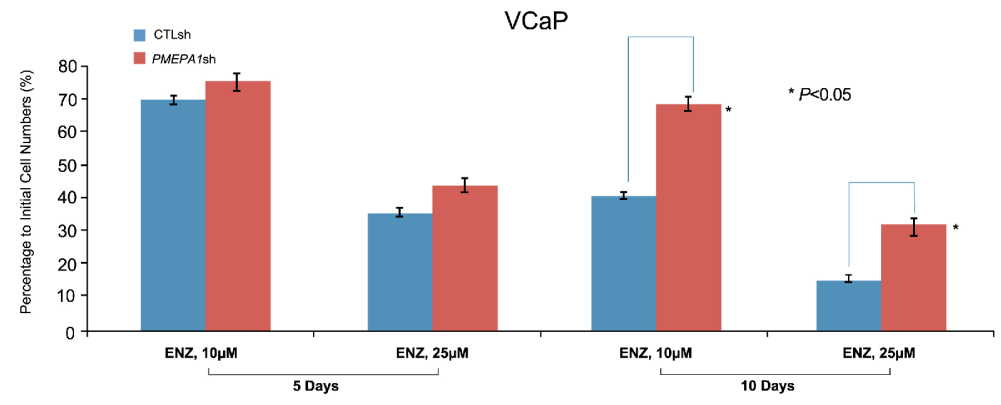

Figure 2: PMEPA1 depletion leads to resistance to AR inhibitors. (A and $B$ ) In contrast to control cells, increased percentages to initial cell numbers $\left(2 \times 10^{5}\right.$ cells $/ 6 \mathrm{~cm}$ dish $)$ were detected in PMEPA1 shRNA harboring LNCaP and VCaP cells in response to enzalutamide (ENZ) treatment at dosages of 10 or $25 \mu \mathrm{M}$ for 5 or 10 days $(p<0.05)$

cell plating efficiency assays (Supplementary Figure 2C-2E). Consistent with these observations, cell cycle analysis showed higher number of cells in S-phase and decreased rate of apoptosis in response to PMEPA1 inhibition (Table 2 and Supplementary Figure 2F). The observed enhanced resistance to AR inhibitors in response to PMEPA1 depletion was consistent with the observed castration resistance of PMEPA1shRNA harboring tumor xenografts. Taken together, these data suggest that loss or decreased PMEPA1 levels confers resistance to AR inhibitors.

\section{$P M E P A 1$ silencing rescues AR inhibition in prostate cancer cells}

In hormone sensitive CaP cells, decreased AR expression levels results in reduced cell growth. However, under this condition, loss of PMEPA1 may supersede the growth effect of AR inhibition by stabilizing AR at protein levels. Thus, we evaluated if inhibition of PMEPA1 can restore $\mathrm{CaP}$ cell growth overriding the inhibition of AR. Indeed, co-transfection of $A R$ and PMEPAI siRNAs in LNCaP cells reversed effects of $A R$ depletion by siRNA on AR protein (Figure 3A). While parental LNCaP cells exhibited severe growth inhibition in response to $A R$ siRNA, simultaneous knockdown of PMEPA1 rescued the growth of cancer cells (Figure 3B). These data further supported that the observed enhanced cell proliferation in response to PMEPA1 depletion was due to elevated AR protein levels and AR signaling.

\section{Disruption of AR and NEDD4 link by loss of PMEPA1}

It was intriguing to note simultaneous increase of NEDD4 and AR in response to PMEPA1 knockdown (Figure 4A). This observation suggested that inhibition of PMEPA1 may disrupt interaction between NEDD4

Table 2: Enzalutamide (ENZ) or bicalutamide (BIC) treated LNCaP cells showed higher percentage of S-phase and lower apoptosis rate in PMEPA1shRNA harboring cells

\begin{tabular}{|l|c|c|c|c|c|c|}
\hline \multirow{2}{*}{ LNCaP cells } & \multicolumn{2}{c}{$\mathbf{0} \boldsymbol{\mu M}$} & \multicolumn{2}{c}{ BIC 25 $\boldsymbol{\mu M}$} & \multicolumn{2}{c}{ ENZ 25 $\boldsymbol{\mu M}$} \\
\cline { 2 - 7 } \multicolumn{1}{c|}{} & S-phase (\%) & Apoptosis (\%) & \multicolumn{1}{c}{ S-phase (\%) } & \multicolumn{1}{c}{ Apoptosis (\%) } & \multicolumn{1}{c}{ S-phase (\%) } & \multicolumn{1}{c}{ Apoptosis (\%) } \\
\hline CTLsh & 14.62 & 6.87 & 8.45 & 18.37 & 6.95 & 23.77 \\
\hline PMEPA1sh & 19.24 & 4.12 & 13.62 & 9.11 & 12.11 & 11.22 \\
\hline$P$ value & 0.0022 & 0.0053 & 0.0168 & 0.007 & 0.023 & 0.0162 \\
\hline
\end{tabular}


A

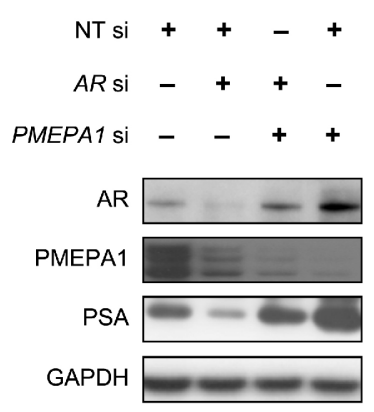

B

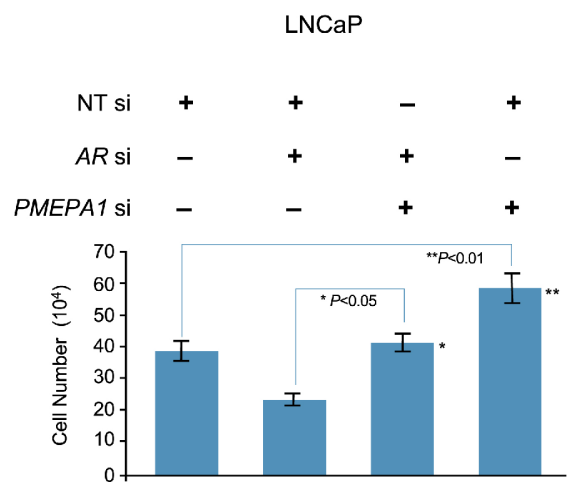

Figure 3: PMEPA1 silencing rescues AR inhibition in prostate cancer cells. (A) Western blot assay shows that $A R$ siRNA (AR si) efficiently decreased protein levels of AR and PMEPA1 when compared to control (NT si). Co-transfection of $A R$ and PMEPA1 siRNA in LNCaP cells reversed effects of the $A R$ depletion by siRNA on AR protein. (B) Cell counting assay reveals that $P M E P A 1$ siRNA (PMEPA1 si) reversed the cell growth inhibition cause by $A R$ siRNA in LNCaP cells $(p<0.01)$.

and AR. This observation was also supported by marked stabilization of AR in LNCaP cells harboring ectopically expressed NEDD4 in the background of PMEPA1 knockdown (Figure 4B). Further, decreases in AR levels in response to ectopic PMEPA1 expression were abolished by NEDD4 knockdown in LNCaP cells (Figure 4C). As expected, the cell growth read out reflected the AR status (Figure 4B and 4C). Conversely, ectopic expression of NEDD4 in PMEPA1 shRNA stable transfectants was refractory to AR biochemical signals and associated features (Figure 4D). These findings established that both PMEPA1 and NEDD4 are essential in AR mediated cell growth.

\section{NEDD4 down-regulates AR protein levels and inhibits cell growth in hormone responsive prostate cancer cells}

Our previous study has revealed that PMEPA1 binds to NEDD4 through the interaction between PY motifs of PMEPA1 and WW domain of human NEDD4 protein [17]. Thus, we evaluated the role of NEDD4 on $\mathrm{CaP}$ cell growth and AR protein levels. Knockdown of NEDD4 by specific siRNA led to strikingly elevated levels of $\mathrm{AR}$ and PSA in $\mathrm{LNCaP}$ and $\mathrm{VCaP}$ cells (Figure 5A). Conversely, ectopic expression of the NEDD4 resulted in decreased levels of AR protein in both LNCaP and VCaP cells (Figure 5B). Further, ectopic expression of NEDD4 resulted in a significant inhibition of cell growth (Figure 5C and 5D). In parallel, we also evaluated the effects MDM2 E3 ligase on AR which has been reported to be involved in AR degradation [15]. As expected, transfection of the MDM2 siRNA, resulted in elevated AR and PSA protein levels in both LNCaP and VCaP cells (Figure 5E and 5F). Taken together, these findings underscored that NEDD4 mediates AR degradation and cell growth inhibition in hormone responsive $\mathrm{VCaP}$ and $\mathrm{LNCaP}$ cells.

\section{NEDD4 facilitates PTEN degradation in PMEPA1 depleted cells}

Genomic deletions and reduced expression of PTEN is frequently noted in $\mathrm{CaPs}$ which correlates with adverse pathologic features [36]. Ubiquitin-mediated degradation of PTEN by NEDD4 has been reported [37, 38]. As noted, above PMEPA1 depletion in CaP cells also resulted in elevated NEDD4 levels. Thus, we reasoned that decreased PMEPA1 may contribute to reduced levels of PTEN, a known NEDD4 target in CaP cells. In VCaP cells harboring wild type PTEN, ectopic expression of NEDD4 downregulates PTEN protein levels (Figure 6A). Moreover, the PMEPA1 knockdown led to elevated levels of AR protein, increased NEDD4 and decreased PTEN protein levels (Figure 6B). These findings suggest that loss of PMEPA1 may contribute to the defects of PTEN and alterations in NEDD4 through AR that is consistent with reported associations of PTEN and AR with CaP progression [36]. Thus, PMEPA1 may in part also affects NEDD4 mediated PTEN degradation in the context of androgen responsive CaP cells. However, NEDD4 can target PTEN independent of AR or PMEPA1. Ectopically expressed PTEN directly interacting with $A R$, has been shown to facilitate $A R$ degradation and to inhibit $\mathrm{AR}$ nuclear translocation in PTEN null LNCaP cells [39]. Due to this reciprocal relationship between $\mathrm{AR}$ and PTEN, we evaluated the cooperative effects of PTEN knockdown and PMEPA1 depletion on cell growth, AR levels and AR transcriptional targets. Transfection of VCaP cells with PMEPA1 or PTEN siRNA resulted in enhanced cell growth, increased levels of AR and its targets, PSA and NEDD4. Cells depleted in both PMEPA1 and PTEN led to further increases in AR and cell growth (Figure 6C and 6D). Cumulatively, loss or reduced expression of PMEPA1 may lead to higher levels of AR and decreased levels of PTEN resulting in a feedforward enhancement in cell growth as shown in simultaneous depletion of PMEPA1 and PTEN. 
A

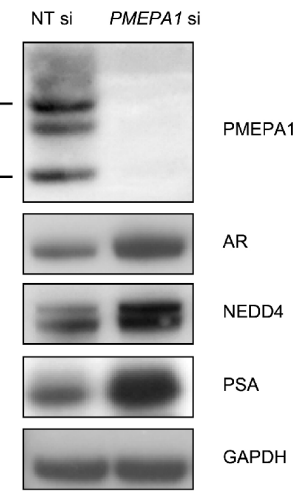

B

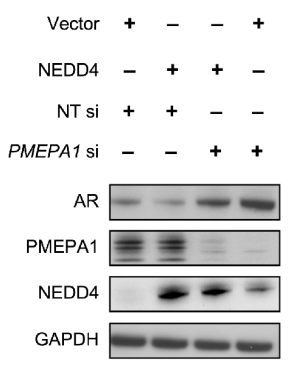

C

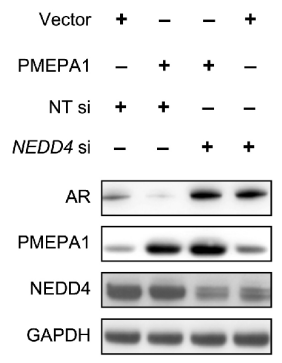

D

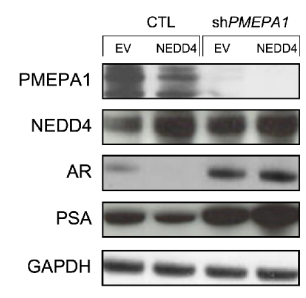

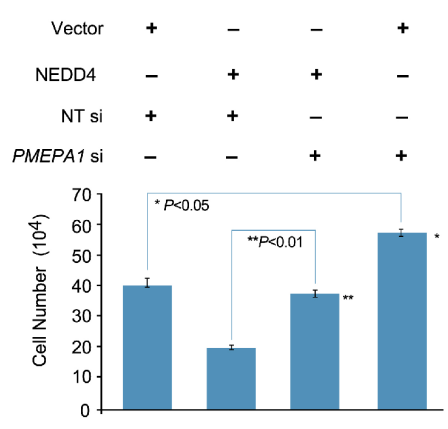
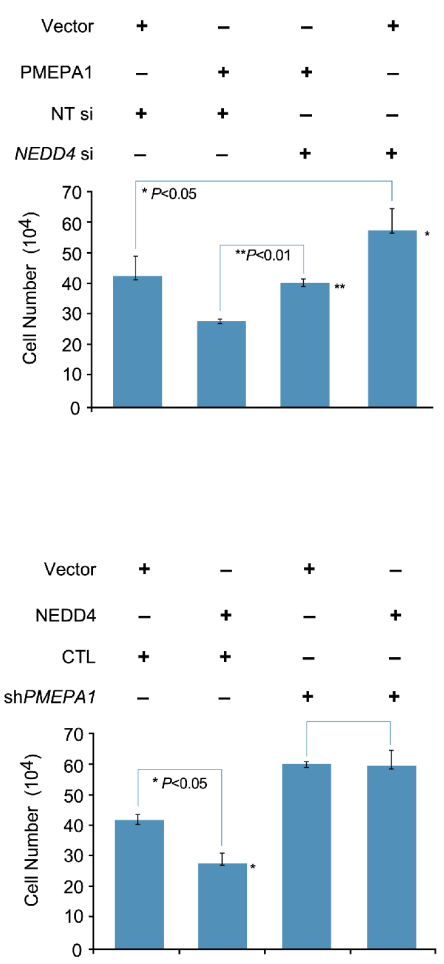

Figure 4: AR and NEDD4 link is disrupted by the loss of PMEPA1. (A) PMEPA1 knockdown increases protein levels of NEDD4, AR and PSA in LNCaP cells as shown by Western blot assay. (B) Knockdown of PMEPAl stabilizes AR (Western blot assay, left panel) and overrides the growth inhibitory effect of ectopically expressed NEDD 4 (cell counting assay, right panel; $p<0.01$ ). (C) $N E D D 4$ siRNA stabilizes AR (Western blot assay, left panel) and reverses the cell growth inhibitory (cell counting assay, right panel) effect of PMEPA1 overexpression $(p<0.01)$. (D) Elevated expression of NEDD4 alone does not affect AR and PSA protein levels (Western blot assay, left panel) or cell growth (cell counting assay, right panel; $p<0.05$ ) in PMEPAlshRNA harboring LNCaP transfectants. 
A

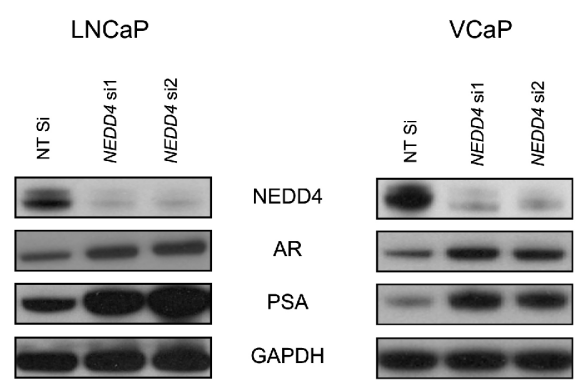

C

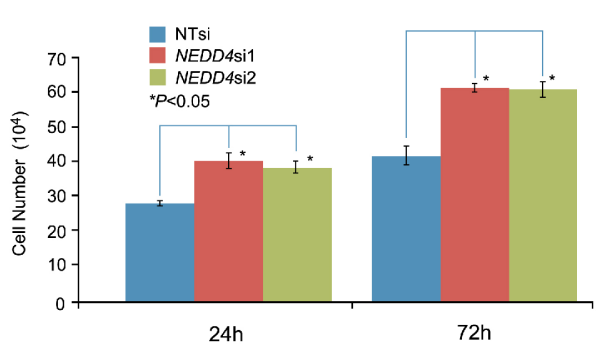

$\mathbf{E}$

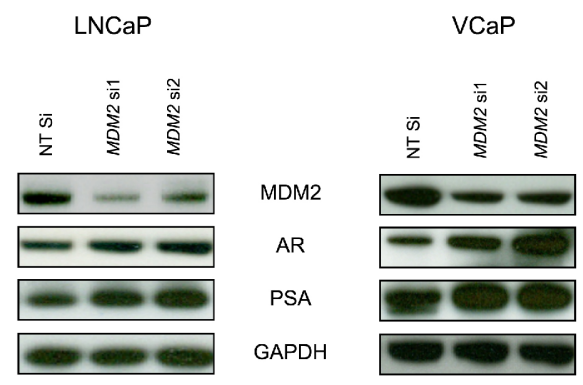

B
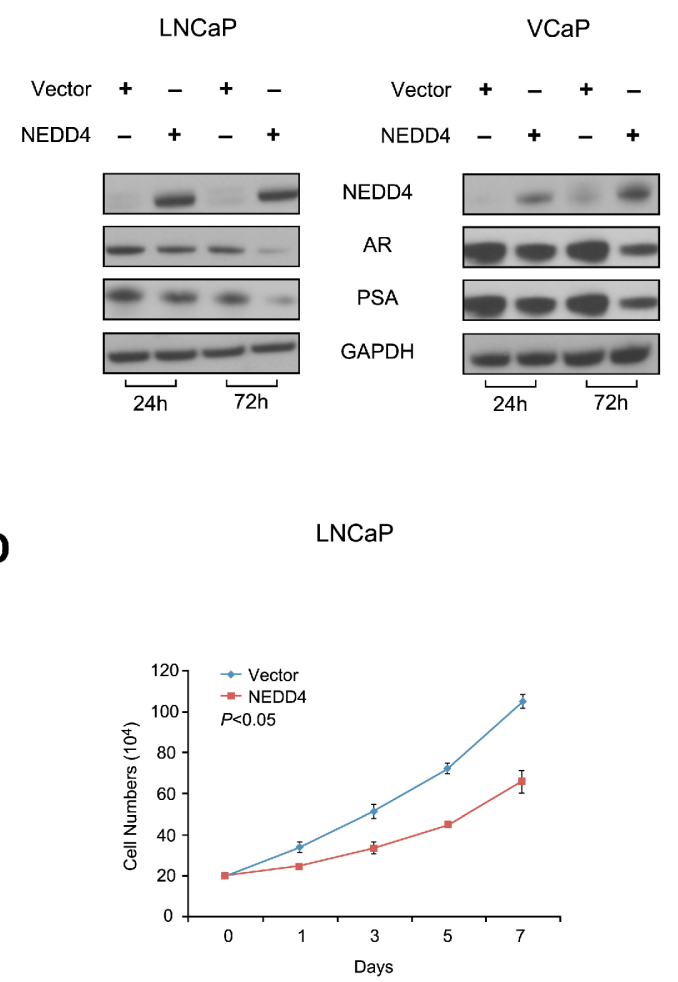

$\mathbf{F}$

LNCaP

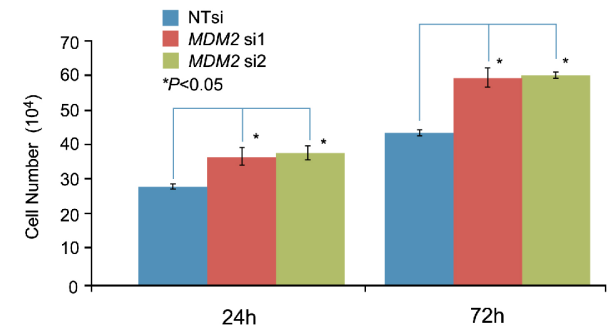

Figure 5: NEDD4 down-regulates of AR protein level and AR signaling, as well as inhibits the growth of LNCaP and VCaP cells. (A) Inhibition of NEDD4 by siRNA (NEDD4 sil and si2) enhances AR and PSA protein levels in LNCaP and VCaP cells. (B) Ectopic expression of NEDD4 decreases AR and PSA in LNCaP and VCaP cells. (C) Cell counting assay reveals that NEDD4 siRNA (NEDD4 sil and si2) increases the growth of LNCaP cells $(p<0.05)$. (D) Conversely, ectopic expression of NEDD4 inhibits the growth of LNCaP cells $(p<0.05)$ as shown by cell counting assay. (E) As expected, MDM2 siRNA (MDM2 sil and si2) also increases AR and PSA protein levels in LNCaP and $\mathrm{VCaP}$ cells.

\section{Higher ratio of $P S A$ to $P M E P A 1$ expression as a surrogate for increased $A R$ function associates with more aggressive prostate cancer cells}

The frequently observed silencing of PMEPA1 in $\mathrm{CaP}$ may result in stabilization of $\mathrm{AR}$, leading to higher levels of $\mathrm{AR}$ and increased AR transcription factor activity. To assess this finding in human $\mathrm{CaP}$ specimens, laser capture micro-dissected matched benign and cancer epithelium derived RNA samples were evaluated for quantitative expression of PMEPA1 and PSA/KLK3 mRNA, a surrogate for AR transcriptional activation function, in frozen radical prostatectomy derived specimens from 82 patients. The experiment revealed a correlation of decreased PMEPA1 mRNA expression with increased PSA/KLK3 expression levels. The ratio of PSA and PMEPAI T/N expression values in each patient was correlated with clinico-pathological characteristics. Higher PSA/PMEPA1 expression ratio significantly associated with multiple indicators of disease progression (tumor differentiation, 

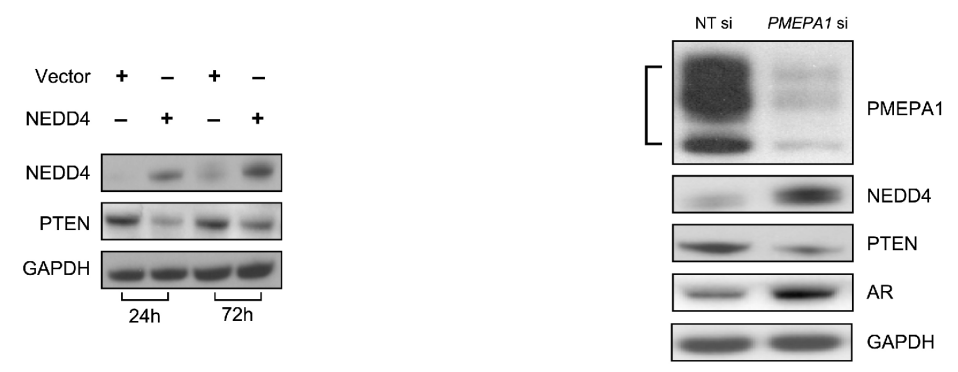

C
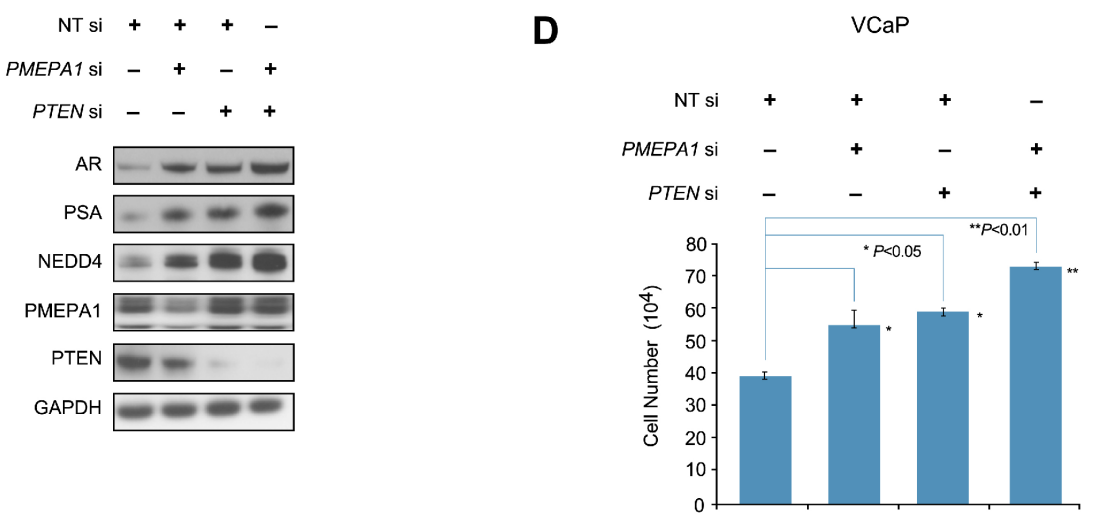

E

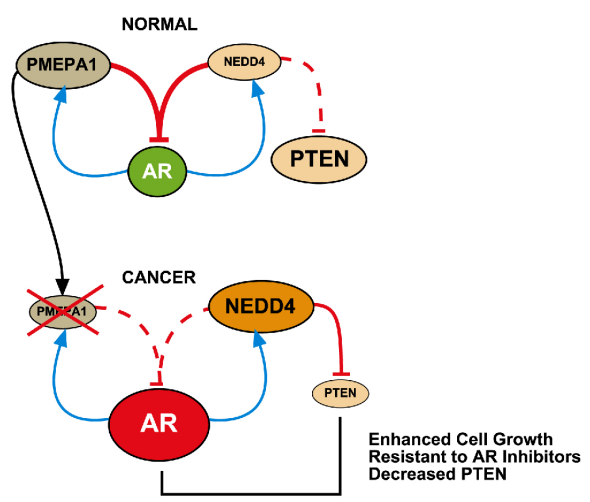

Figure 6: NEDD4 facilitates PTEN degradation in PMEPA1 depleted cells. (A) Western blot assay shows that overexpression of NEDD4 decreases PTEN in VCaP cells at 24 and 72 hours post transfection. (B) Inhibition of PMEPA1 by siRNA upregulates NEDD4 and AR, and results in decreases in PTEN protein levels in VCaP cells. (C) Simultaneous knockdown of PMEPA1 and PTEN leads to elevated AR and PSA levels and (D) enhanced cell growth $(p<0.01)$. (E) Model depicting three major consequences of PMEPA1 depletion in $\mathrm{CaP}$ cells such as increased cell growth, resistances to AR inhibitors and decrease PTEN protein level.

margin status, extracapsular extension and seminal vesicle invasion (Table 3). Thus, increased AR function as reflected by increased PSA $/ K L K 3$ expression along with reduced PMEPA1 expression associate with progressive $\mathrm{CaP}$.

\section{DISCUSSION}

It is well recognized that the alterations (gain or loss) of the AR mediated signaling, play critical roles in the development and progression of CaP. While AR has been extensively studied for alterations of its structure, expression and transcriptional functions in $\mathrm{CaP}$, defects of the $\mathrm{AR}$ protein degradation in $\mathrm{CaP}$ warrant careful evaluations. Better understanding of mechanisms controlling AR protein levels may also help in developing new therapeutic strategies focusing on $\mathrm{AR}$ degradation in a majority of metastatic $\mathrm{CaP}$ associating with the gain of AR function.

Cumulative data underscore the association of reduced or absent PMEPA1 expression in CaP cells with increased AR due to impaired AR degradation [17-19]. 
Table 3: Assessment of AR activity by PSA/KLK3 gene expression relative to PMEPA1 showed association of increased $\mathrm{PSA}(K L K 3) / P M E P A 1$ ratios with poor clinicopathologic features

\begin{tabular}{|c|c|c|c|c|}
\hline \multicolumn{2}{|c|}{ Clinicopathologic Characteristics } & \multicolumn{3}{|c|}{ Log ratio PSA(KLK3)/PMEPA1 T/N } \\
\hline & & $N$ & Mean (SD) & $P$ value \\
\hline \multirow{2}{*}{$\begin{array}{l}\text { LCM Tumor } \\
\text { Differentiation }\end{array}$} & Well & 68 & $0.52(1.37)$ & \multirow[t]{2}{*}{0.0218} \\
\hline & Poorly & 14 & $1.52(1.80)$ & \\
\hline \multirow[t]{2}{*}{ Margin status } & Negative & 66 & $0.5(1.4)$ & \multirow[t]{2}{*}{0.0293} \\
\hline & Positive & 16 & $1.3(1.6)$ & \\
\hline \multirow{3}{*}{$\begin{array}{l}\text { Extra Capsular } \\
\text { Extensions (ECE) }\end{array}$} & Negative (pT2) & 47 & $0.4(1.4)$ & \multirow[t]{2}{*}{0.0251} \\
\hline & Positive (pT2-3) & 34 & $1.1(1.5)$ & \\
\hline & & \multicolumn{3}{|c|}{$\operatorname{PSA}(K L K 3) / P M E P A 1(\mathrm{~T})$ Ratio Fold Change } \\
\hline \multirow{2}{*}{$\begin{array}{l}\text { Seminal Vesicle } \\
\text { (SV) Invasion }\end{array}$} & Negative & 71 & 0.006 & \multirow[t]{2}{*}{0.0063} \\
\hline & Positive & 11 & 2.828 & \\
\hline
\end{tabular}

However, the impact of attenuated PMEPA1 expression in the interface of NEDD4, AR and PTEN in CaP biology and the effects on therapeutic AR inhibitors has not been examined before. Three major consequences of loss of PMEPA1 in CaP are summarized in the working model (Figure 6E). First, PMEPA1 knockdown accelerated the growth of LNCaP tumors in athymic nude mouse and in cell culture models. Second, PMEPA1 knockdown resulted in enhanced resistance to both enzalutamide and bicalutamide inhibitors of AR. Third, we noted that PMEPA1 silencing increased NEDD4-mediated downregulation of PTEN, further enhancing the growth of $\mathrm{CaP}$ cells. In agreement with cell culture models, in human $\mathrm{CaP}$ specimens decreased PMEPA1 expression correlated with increased AR transcriptional function as measured by PSA/KLK3 mRNA expression.

One of the new findings of this study is the androgen induction of NEDD4 E3 ligase that may impose additional level of hormonal control on AR and PTEN degradation (Supplementary Figure 3A-3C). Previous studies reported androgen induction of $N E D D 4 L$ transcripts [40]. Informatics analysis of regulatory elements in the NEDD4 gene locus indicated the presence of a cluster of androgen responsive elements (Supplementary Figure 3D).

Of note, this study also provides novel findings of cooperation between PMEPA1 and PTEN defects leading to enhanced $\mathrm{CaP}$ cell growth. While PMEPA1 depletion uncouples NEDD4 from AR degradation, increased levels of NEDD4 remains available for PTEN degradation, leading to simultaneous gain of AR and loss of PTEN in $\mathrm{CaP}$ cells. Evaluating the mechanism of PMEPA1 silencing in previous studies we have shown that methylation of the first intronic DNA region of PMEPA1 gene is a prevalent mechanism of PMEPA1 silencing in human prostate $\mathrm{CaP}$ [41]. In conclusion, this study highlights that silencing of PMEPAI accelerates the growth of $\mathrm{CaP}$ cells through $\mathrm{AR}$, NEDD4 and PTEN. Taken together, these data provide support for the dual impact of PMEPA1 restoration as a complementary approach in correcting for AR and PTEN defects in $\mathrm{CaP}$ and highlights biomarker potential of monitoring PMEPA1 during androgen ablation therapy.

\section{MATERIALS AND METHODS}

\section{Cell culture and related reagents}

LNCaP and VCaP cells (from ATCC, Manassas VA) were maintained in RPMI 1640 and DMEM growth medium, respectively, supplemented with $10 \%$ fetal bovine serum (FBS). For the androgen-depletion experiments, cells were grown in $10 \%$ charcoal stripped FBS supplemented cell growth medium for 5 days. Subsequently, cells were treated with various dosages $(0 \mathrm{nM}, 0.1 \mathrm{nM}, 1.0 \mathrm{nM}$ and $10.0 \mathrm{nM})$ of synthetic androgen R1881 (Cat\#NLP005005MG, Perkin Elmer Life Science, Waltham, MA) for indicated time. Hexamethrine bromide (Cat\#H9268-5G) and puromycin (Cat\#A1113802) used for selection PMEPA1 shRNA lentivirus transfectants were purchased from Sigma-Aldrich (St. Louis, MO).

\section{Plasmids, small interfering (si) RNAs and shRNA lentiviruses}

The mammalian plasmids used were as follows: pcDNA3.1 as vehicle control, pcDNA3.1-PMEPA1, pCMV-AR (wild-type) and pCMV-AR (mutant T877A) were described before $(15,37)$; pCMV-XL4 (vehicle control, Cat\#PCMV6XL4) and pCMV-XL4-NEDD4 (Cat\#SC107811) were purchased from OriGene (Rockville, MD). The siRNAs for various genes were all purchased from Dharmacon (Lafayette, CO). Each gene of interest was targeted with two different siRNAs (Supplementary 4A-4C) using optimal concentrations that were determined in pilot assays: PMEPA1 siRNA1: GTTATCACCACGTATATA; 
PMEPA1 siRNA2: 5'-GCATCAGCGCCACGTGCTA-3'; NEDD4 siRNA1: 5'-TGCAGAACAGGCTGAGGAA-3'; NEDD4 SiRNA2 ATGAAACTTCGCCGAGCAA; $A R$ SiRNA1: 5'-GCAAAGGTTCTCTGCTAGA-3'; AR siRNA2: 5'-TCGAGGCCCTGTAACTTG-3'; MDM2 SiRNA1: 5'-GCCACAAATCTGATAGTA-3'; MDM2 SiRNA2: 5'-GAAGTTATTAAAGTCTGTT-3'; PTEN SiRNA1: 5'-GTATAGAGCGTGCAGATAA-3'; PTEN siRNA2: 5'-GTTAGCAGAAACAAAAGGAGATATCAA-3'. The co-transfection was conducted with PMEPA1 siRNA1, $A R$ siRNA2 and PTEN siRNA2. The non-targeting control siRNA pool (Cat\#D-001206-13-50) was also purchased from Dharmacon (Lafayette, CO). The Mission shRNA lentiviral transduction particles (Non-Target, Cat\#SHC002V and PMEPA1, Cat\#SHCLNV and Clone ID: TRCN0000272494) were purchased from Sigma-Aldrich (St. Louis, MO). The targeting sequence of PMEPA1 shRNA1 was 5'-GAGCAAAGAGAAGGATAAACA-3', and PMEPA1 shRNA2 was 5'-GAGTTTGTTCAGATCATCATC-3'. The transfection of plasmids or small interfering RNA were performed following the standard protocol of lipofectamine 2000 (Life Technology, Carlsbad, CA). The infection of mission shRNA lentiviruses transduction particles and selection of positive colonies were performed as recommended by the supplier.

\section{Cell counting assays}

The CaP cells (LNCaP and VCaP cells) were seeded in $6 \mathrm{~cm}$ culture dishes at the density of $2 \times 10^{5}$ cells $/$ dish. Cells were grown at $37^{\circ} \mathrm{C}, 5 \% \mathrm{CO}_{2}$ for 2 days followed by transfection with plasmids (pcDNA3.1, pcDNA3.1PMEPA1, pCMV-XL4, pCMV-XL4-NEDD4 or pCMVXL4- $A R$ ) or siRNA (non-targeting, PMEPA1, AR, NEDD4 or PTEN siRNA) using lipofectamine 2000 procedure. The AR inhibitors treatment experiments were conducted with PMEPA1 shRNA1 harboring LNCaP and VCaP cells. The transfected cells or the stable transfectants were harvested at indicated time points post-transfection with $0.25 \%$ trypsin plus EDTA (Life Technology, Carlsbad, CA). Cells were re-suspended into $10 \mathrm{ml}$ regular medium, and $10 \mu \mathrm{l}$ of single cell suspension was applied to hemocytometer for cell counting with trypan blue (Cat\#72-57-1, SigmaAldrich, St. Louis, MO).

\section{Cell cycle analysis by fluorescence activated cell sorting}

Ten million cells of PMEPA1 shRNA transfectant $\mathrm{LNCaP}$ or $\mathrm{VCaP}$ cells were harvested by centrifugation at $180 \times \mathrm{g}$ for $5 \mathrm{~min}$. Cells were resuspended in $300 \mu \mathrm{l}$ PBS (pH 7.4), and $5 \mathrm{ml}$ methanol was added drop-wise and vortexed at low speed. The fixed cells were incubated in methanol at $-20^{\circ} \mathrm{C}$ for $30 \mathrm{~min}$. After removing the methanol cells were stained by propidium iodide dye (Sigma-Aldrich, St. Louis, MO) at final concentration of
$60 \mu \mathrm{g} / \mathrm{ml}$ containing $50 \mu \mathrm{g} / \mathrm{ml}$ RNaseA in PBS at room temperature (RT) for $30 \mathrm{~min}$ and were processed for fluorescence-activated cell sorting (FACS) analysis.

\section{Western blot analysis}

The cells were harvested with M-PER mammalian protein extraction reagent (Cat\#78501, Thermo Scientific, Rockford, IL) supplemented with protease inhibitor mixture (Cat\#P8340, Roche Applied Science, Indianapolis, IN) and phosphatase inhibitor mixture (Cat\#P-0044, Sigma-Aldrich, St. Louis, MO). Cell lysates were analyzed using Western blot procedures described in details in the Supplementary Methods.

\section{Antibodies}

Antibodies used for Western blot assay were as follows: anti-AR rabbit polyclonal antibody (N-20, Cat\#sc-816, Santa Cruz Biotechnology, Santa Cruz, CA); anti-PMEPA1 mouse monoclonal antibody (2A12, Cat\#H00056937-M01, ABNOVA, Taiwan); anti-NEDD4 rabbit polyclonal antibody (Cat\#07-049, Millipore, Billerica, MA); anti-PTEN mouse monoclonal antibody (Cat\#MS-1601-S0, Lab Vision, Fremont, CA), antiPSA rabbit polyclonal antibody (Cat\#A05662, Dako, Denmark); anti-GAPDH polyclonal antibody (FL335, Cat\#sc-25778, Santa Cruz Biotechnology, Santa Cruz, CA); and horseradish peroxidase (HRP)-conjugated anti-rabbit and anti-mouse TrueBlot antibodies (Cat\#188816, Cat\#18-8817-33, eBioscience, San Diego, CA). Antibodies for immunohistochemistry (IHC) assay were: anti-PMEPA1 antibody mentioned above, anti-AR mouse monoclonal antibody (M3562, DAKO, Denmark), antiPSA rabbit polyclonal antibody (Cat\#A0562, DAKO, Denmark), biotinylated horse anti-mouse antibody (Cat\# BA-2000, Vector, Burlingame, CA), biotinylated goat antirabbit antibody (Cat\# BA-1000, Vector, Burlingame, CA) and VIP peroxidase (HRP) substrate kit (Cat\# SK-4600, Vector, Burlingame, CA).

\section{Athymic nude mouse tumorigenicity assay}

Ten weeks old athymic Ncr-nu/nu mice (National Cancer Institute, Frederick, MD) were evaluated in tumor xenografts derived from PMEPAI shRNA LNCaP and control-shRNA LNCaP cells. Mice were maintained under pathogen-free conditions in accordance with established $\mathrm{NIH}$ guideline under the animal protocol approved by Uniformed Services of University of the Health Sciences, Institutional Animal Use and Care Committee. A total of 40 athymic nude mice were subdivided into two groups: control-shRNA LNCaP and PMEPA1-shRNA LNCaP with 20 mice in each group. Transfectant cells were resuspended in RPMI1640 medium mixed with 50\% matrigel (Cat\#354234, BD Biosciences, Bedford, MA). Each mouse received $4 \times 10^{6}$ cells in $200 \mu \mathrm{l}$ medium by subcutaneous injection 
to right frank side. Injected mice were monitored for tumor formation twice per week post injection. The end point of the experiment represented tumor diameter of $1.5 \mathrm{~cm}$ or 6 weeks after xenograft tumor formation. The excised tumor tissue was fixed in 10\% formalin, paraffin-embedded and sectioned for $5 \mu \mathrm{m}$ slides for Hematoxylin and Eosin stain and immunohistochemistry (IHC) staining for PMEPA1, AR and PSA. Total 10 foci with $20 \mathrm{X}$ magnification were randomly examined from each IHC staining slides for quantitative analysis with ImageJ software (version 1.48, National Institutes of Health, Bethesda, MD). The IHC procedures were described in details in the Supplementary Methods.

\section{Quantitative RT-PCR}

Quantitative RT-PCR was performed using RNA laser capture microdissected matched normal and prostate tumor specimens as described in Sterbis et al [42]. PCR primers and Taqman probes were as follows: PMEPA1, 5'-CATGATCCCCGAGCTGCT-3' (forward), 5'-TGATCTGAACAAACTCCAGCTCC-3' (reverse), and 6FAM-5'-AGGCGGACAGTCTCCTGCGAAACC3'-TAMRA (probe); PSA/KLK3, 5'-CCCACTG CATCAGGAACAAA-3' (forward), 5'-GAGCGGGTGTG GGAAGCT-3' (reverse), and 6FAM-5'-ACACAGGCCAG GTATTTCAGGTCAGCC-3'-TAMRA (probe). The expression of $G A P D H$ served as an endogenous control measured in the same tube as the target gene (GAPDH control mix, Applied Biosystems). Target gene expression in each sample was normalized to $G A P D H$. Results were plotted as average $\mathrm{Ct}$ (cycle threshold) values of duplicate samples. Relative gene expression level was presented as $\mathrm{dCt}$ in tumor versus matched benign cells, where $\mathrm{dCt}$ represents normalized $\mathrm{Ct}$ value of target genes to GAPDH. Statistical analysis of quantitative expression of $P M E P A 1$ and PSA mRNA was evaluated in 82 patients (164 tumor and matched normal samples). The sample size $(N=82)$ was determined by statistical power calculation $(90 \%$ power with 0.05 alpha, two sided Chi-square test). Distributions of other clinico-pathological variables were examined by using Chi-square or Fisher exact tests.

\section{Statistical analysis}

If not otherwise stated, significant was calculated utilizing an unpaired $t$-test. Data are calculated as mean \pm SEM or + SEM.

\section{ACKNOWLEDGMENTS}

This study was in part supported by the National Institute of Health grant, CA106653 to SS and AD and Center for Prostate Disease Research, Uniformed Services University HU0001-10-2-0002 to DGM. The authors thank Dr. Yongmei Chen for the statistical analysis of gene expression data, Ms. Lakshmi Ravindranath for providing cell culture models, Ms. London Toney for the laboratory management support and Mr. Stephen Doyle for his assistance with the art and graphics. The views expressed in this manuscript are those of the authors and do not reflect the official policy of the Department of the Army, Department of Defense or the US Government.

\section{CONFLICTS OF INTEREST}

No potential conflicts of interest were disclosed.

\section{SOURCES OF SUPPORT}

This study was in part supported by the NIH grant, CA106653 to SS and AD and Center for Prostate Disease Research, Uniformed Services University HU0001-10-20002 to DGM.

\section{REFERENCES}

1. Schmidt LJ, Tindall DJ. Androgen receptor: past, present and future. Curr Drug Targets. 2013; 14:401-407.

2. Yuan X, Cai C, Chen S, Yu Z, Balk SP. Androgen receptor functions in castration-resistant prostate cancer and mechanisms of resistance to new agents targeting the androgen axis. Oncogene. 2014; 33:2815-2825.

3. Izumi K, Mizokami A, Lin WJ, Lai KP, Chang C. Androgen receptor roles in the development of benign prostate hyperplasia. Am J Pathol. 2013; 182:1942-1949.

4. Richter E, Masuda K, Cook C, Ehrich M, Tadese AY, Li H, Owusu A, Srivastava S, Dobi A. A role for DNA methylation in regulating the growth suppressor PMEPA1 gene in prostate cancer. Epigenetics. 2007; 2:100-109.

5. Dobi A, Sreenath T, Srivastava S. Androgen-Dependent Oncogenic Activation of ETS Transcription Factors by Recurrent Gene Fusions in Prostate Cancer: Biological and Clinical Implications. In: Wang Z. Androgen-Responsive Genes in Prostate Cancer- Regulation, Function and Clinical Applications. New York: Springer Science 2013:307-328.

6. Siegel R, Ma J, Zou Z, Jemal A. Cancer statistics, 2014. CA Cancer J Clin. 2014; 64:9-29.

7. Kantoff PW, Mohler JL. New developments in the management of prostate cancer. J Natl Compr Canc Netw. 2013; $11: 653-657$.

8. Tennakoon JB, Shi Y, Han JJ, Tsouko E, White MA, Burns AR, Zhang A, Xia X, Ilkayeva OR, Xin L, Ittmann MM, Rick FG, Schally AV, et al. Androgens regulate prostate cancer cell growth viaan AMPK-PGC-1amediated metabolic switch. Oncogene. 2014; 33:5251-5261.

9. Wong YN, Ferraldeschi R, Attard G, de Bono J. Evolution of androgen receptor targeted therapy for advanced prostate cancer. Nat Rev Clin Oncol. 2014; 11:365-376.

10. Bastos DA, Dzik C, Rathkopf D, Scher HI. Expanding androgen- and androgen receptor signaling-directed therapies for castration-resistant prostate cancer. Oncology (Williston Park). 2014; 28:693-699. 
11. Fahrenholtz CD1, Rick FG, Garcia MI, Zarandi M, Cai RZ, Block NL, Schally AV, Burnstein KL. Preclinical efficacy of growth hormone-releasing hormone antagonists for androgen-dependent and castration-resistant human prostate cancer. Proc Natl Acad Sci USA. 2014; 111:1084-1089.

12. Li B, Lu W, Chen Z. Regulation of Androgen Receptor by E3 Ubiquitin Ligases: for More or Less. Receptors Clin Investig. 2014; 1. doi: 10.14800/rci.122.

13. van der Steen T, Tindall DJ, Huang H. Posttranslational modification of the androgen receptor in prostate cancer. Int J Mol Sci. 2013; 14:14833-14859.

14. Gregory CW, Johnson RT, Jr., Mohler JL, French FS, Wilson EM. Androgen receptor stabilization in recurrent prostate cancer is associated with hypersensitivity to low androgen. Cancer Res. 2001; 61:2892-2898.

15. Lin HK, Wang L, Hu YC, Altuwaijri S, Chang C. Phosphorylation-dependent ubiquitylation and degradation of androgen receptor by Akt require Mdm2 E3 ligase. EMBO J. 2002; 21:4037-4048.

16. Xu LL, Shanmugam N, Segawa T, Sesterhenn IA, McLeod DG, Moul JW, Srivastava S. A novel androgenregulated gene, PMEPA1, located on chromosome 20q13 exhibits high level expression in prostate. Genomics. 2000; 66:257-263.

17. Xu LL, Shi Y, Petrovics G, Sun C, Makarem M, Zhang W, Sesterhenn IA, McLeod DG, Sun L, Moul JW, Srivastava S. PMEPA1, an androgen-regulated NEDD4-binding protein, exhibits cell growth inhibitory function and decreased expression during prostate cancer progression. Cancer Res. 2003; 63:4299-4304.

18. Masuda K, Werner T, Maheshwari S, Frisch M, Oh S, Petrovics G, May K, Srikanta V, Srivatava S, Dobi A. Androgen receptor binding sites identified by a GREF GATA model. J Mol Biol. 2005; 353:763-771.

19. Li H, Xu LL, Masuda K, Raymundo E, McLeod DG, Dobi A, Srivastava S. A feedback loop between the androgen receptor and a NEDD4-binding protein, PMEPA1, in prostate cancer cells. J Biol Chem. 2008; 283:28988-28995.

20. Litvinov IV, Vander Griend DJ, Antony L, Dalrymple S, De Marzo AM, Drake CG, Isaacs JT. Androgen receptor as a licensing factor for DNA replication in androgensensitive prostate cancer cells. Proc Natl Acad Sci USA. 2006; 103:15085-15090.

21. D'Antonio JM, Vander Griend DJ, Isaacs JT. DNA licensing as a novel androgen receptor mediated therapeutic target for prostate cancer. Endocr Relat Cancer. 2009; 16:325-332.

22. Barbieri CE, Baca SC, Lawrence MS, Demichelis F, Blattner M, Theurillat JP, White TA, Stojanov P, Van Allen E, Stransky N, Nickerson E, Chae SS, Boysen G, et al. Exome sequencing identifies recurrent SPOP, FOXA1 and MED12 mutations in prostate cancer. Nat Genet. 2012; 44:685-689.
23. An J, Wang C, Deng Y, Yu L, Huang H. Destruction of full-length androgen receptor by wild-type SPOP, but not prostate-cancer-associated mutants. Cell Rep. 2014; 6:657-669.

24. Jolliffe CN, Harvey KF, Haines BP, Parasivam G, Kumar S. Identification of multiple proteins expressed in murine embryos as binding partners for the WW domains of the ubiquitin-protein ligase Nedd4. Biochem J. 2000; 35:557-565.

25. Yang B, Kumar S. Nedd4 and Nedd4-2: closely related ubiquitin-protein ligases with distinct physiological functions. Cell Death Differ. 2010; 17:68-77.

26. An H, Krist DT, Statsyuk AV. Crosstalk between kinase and Nedd4 family ubiquitin ligases. Mol Biosyst. 2014; 10:1643-1657.

27. Rae FK, Hooper JD, Nicol DL, Clements JA. Characterization of a novel gene, STAG1/PMEPA1 upregulated in renal cell carcinoma and other solid tumors. Mol Carcinog. 2001; 32:44-53.

28. Giannini G, Ambrosini MI, Di Marcotullio L, Cerignoli F, Zani M, MacKay AR, Screpanti I, Frati L, Gulino A. EGFand cell-cycle-regulated STAG1/PMEPA1/ERG1.2 belongs to a conserved gene family and is overexpressed and amplified in breast and ovarian cancer. Mol Carcinog. 2003; 38:188-200.

29. Brunschwig EB, Wilson $\mathrm{K}$, Mack D, Dawson D, Lawrence E, Willson JK, Lu S, Nosrati A, Rerko RM, Swinler S, Beard L, Lutterbaugh JD, Willis J, et al. PMEPA1, a transforming growth factor-beta-induced marker of terminal colonocyte differentiation whose expression is maintained in primary and metastatic colon cancer. Cancer Res. 2003; 63:1568-1575.

30. Watanabe $\mathrm{Y}$, Itoh $\mathrm{S}$, Goto $\mathrm{T}$, Ohnishi $\mathrm{E}$, Inamitsu $\mathrm{M}$, Itoh F, Satoh K, Wiercinska E, Yang W, Shi L, Tanaka A, Nakano N, Mommaas AM, et al. TMEPAI, a transmembrane TGF-beta-inducible protein, sequesters Smad proteins from active participation in TGF-beta signaling. Mol Cell. 2010; 37:123-134.

31. Singha PK, Yeh IT, Venkatachalam MA, Saikumar P. Transforming growth factor-beta (TGF-beta)-inducible gene TMEPAI converts TGF-beta from a tumor suppressor to a tumor promoter in breast cancer. Cancer Res. 2010; 70:6377-6383.

32. Hu Y, He K, Wang D, Yuan X, Liu Y, Ji H, Song J. TMEPAI regulates EMT in lung cancer cells by modulating the ROS and IRS-1 signaling pathways. Carcinogenesis. 2013; 34:1764-1772.

33. Vo Nguyen TT, Watanabe Y, Shiba A, Noguchi M, Itoh S, Kato M. TMEPAI/PMEPA1 enhances tumorigenic activities in lung cancer cells. Cancer Sci. 2014; 105:334-341.

34. Hirokawa YS, Takagi A, Uchida K, Kozuka Y, Yoneda M, Watanabe M, Shiraishi T. High level expression of STAG1/ PMEPA1 in an androgen-independent prostate cancer PC3 subclone. Cell Mol Biol Lett. 2007; 12:370-377. 
35. Liu R, Zhou Z, Huang J, Chen C. PMEPA1 promotes androgen receptor-negative prostate cell proliferation through suppressing the Smad3/4-c-Myc-p21 Cip1 signaling pathway. J Pathol. 2011; 223:683-694.

36. Wang X, Trotman LC, Koppie T, Alimonti A, Chen Z, Gao Z, Wang J, Erdjument-Bromage H, Tempst P, Cordon-Cardo C, Pandolfi PP, Jiang X. NEDD4-1 is a proto-oncogenic ubiquitin ligase for PTEN. Cell. 2007; 128:129-139.

37. Trotman LC, Wang X, Alimonti A, Chen Z, TeruyaFeldstein J, Yang H, Pavletich NP, Carver BS, CordonCardo C, Erdjument-Bromage H, Tempst P, Chi SG, Kim HJ, et al. Ubiquitination regulates PTEN nuclear import and tumor suppression. Cell. 2007; 128:141-156.

38. Beltran H, Rubin MA. New strategies in prostate cancer: translating genomics into the clinic. Clin Cancer Res. 2013; 19:517-523.

39. Lin HK, Hu YC, Lee DK, Chang C. Regulation of androgen receptor signaling by PTEN (phosphatase and tensin homolog deleted on chromosome 10) tumor suppressor through distinct mechanisms in prostate cancer cells. Mol Endocrinol. 2004; 18:2409-2423.

40. Qi H, Grenier J, Fournier A, Labrie C. Androgens differentially regulate the expression of NEDD4L transcripts in LNCaP human prostate cancer cells. Mol Cell Endocrinol. 2003; 210:51-62.

41. Sharad S, Ravindranath L, Haffner MC, Li H, Yan W, Sesterhenn IA, Chen Y, Ali A, Srinivasan A, McLeod DG, Yegnasubramanian S, Srivastava S, Dobi A, et al. Methylation of the PMEPA1 gene, a negative regulator of the androgen receptor in prostate cancer. Epigenetics. 2014; 9:918-927.

42. Sterbis JR, Gao C, Furusato B, Chen Y, Shaheduzzaman S, Ravindranath L, Osborn DJ, Rosner IL, Dobi A, McLeod DG, Sesterhenn IA, Srivastava S, Cullen J, et al. Higher expression of the androgen-regulated gene PSA/HK3 mRNA in prostate cancer tissues predicts biochemical recurrence-free survival. Clin Cancer Res. 2008; 14:758-763. 